THE RESEARCH INSTITUTE OF INDUSTRIAL ECONOMICS

WORKING PAPER No. 476, 1997

SOCIAL NORMS AND ECONOMIC INCENTIVES IN THE WELFARE STATE

By ASSAR LINDBECK, STEN NYberg AND JÖRGEN W. WEIBULL 


\title{
Social Norms and Economic Incentives in the Welfare State*
}

\author{
Assar Lindbeck ${ }^{\dagger}$ Sten Nyberg ${ }^{\ddagger}$ and Jörgen W. Weibull ${ }^{\S}$
}

April 15, 1997

\begin{abstract}
This paper analyzes the interplay between social norms and economic incentives in the context of work decisions in the modern welfare state. We assume that to live off one's own work is a social norm, and that the larger the population fraction adhering to this norm, the more intensely it is felt by the individual. Individuals face two choices, one economic, whether to work or live off public transfers, and one political, how large the transfer should be. The model highlights certain factors determining the size of the welfare state. Doc: $\ln w \%$.tex.
\end{abstract}

${ }^{*}$ The authors wish to thank Sören Blomquist, Avinash Dixit, Tore Ellingsen, Nils Gottfries, Joseph Harrington, Ulf Jakobson, Yeongjae Kang, Yvan Lengwiler, John Roemer, Peter Sorensen, Richard Swedberg, Karl Wärneryd, and the participants in seminars at Cornell and Humboldt University, the Universities of Uppsala and Stockholm and at the IUI (The Research Institute of Industrial Economics) for helpful comments. We also thank David Sundén for excellent assistance with computer illustrations.

${ }^{\dagger}$ The Institute for International Economic Studies, Stockholm University, and the IUI.

FDepartment of Economics, Stockholm University, and the IUI.

$\S$ Department of Economics, Stockholm School of Economics, and the IUT. 


\section{Introduction}

Both economic incentives and social norms influence individual behavior. While sociologists have emphasized social norms, economists have focused on economic incentives. ${ }^{1}$ Most likely the different approaches reflect the different subject matters dealt with in the two disciplines. Social norms are likely to play a major role in certain decisions that people make, while other decisions seem to be driven primarily by economic incentives. For some decisions, however, both social norms and economic incentives appear to be involved. We therefore feel that it is important to take both the economic and the sociologic paradigms seriously.

This paper is an attempt to bring together social norms and economic incentives. More precisely, we extend the traditional economic model of individual preferences to encompass social norms in an analytically tractable way. The purpose is to enable an analysis of, on the one hand, economic decisions where individuals take the adherence to the social norm in society as given, and, on the other hand, political decisions where individuals anticipate the influence of the chosen policy on this adherence.

We have chosen to develop this point in a specific context, namely economic and political decisions concerning work and benefits in the modern welfare state. The relevance of this topic is evident from the fact that such benefits have increased dramatically in recent decades and now constitute a large fraction of the national income in most OECD countries, in particular in western Europe. Examples of such benefit systems are social assistance ("welfare" in US terminology), early retirement, sickness benefits, paid parental leave and unemployment benefits. About 45 percent of the adult Swedish population, including old-age pensioners, and about 25 percent excluding this group, is at the present time basically financed in this way. ${ }^{2}$ These figures do not include individuals who are only partially financed by social benefits such as child allowances, day care subsidies and housing allowances. Similar, though somewhat lower, numbers are found in some other west-European countries such as Belgium and Denmark. In most countries the number of welfare state beneficiaries has risen dramatically in recent decades. ${ }^{3}$

We feel that individual decisions concerning work belongs to the class of decisions where both economic incentives and social norms play a role. To quote Jon Elster ([8], p. 121), "The work place is a hotbed of norm-guided action. ... There is a social norm against living off other people and a corresponding

\footnotetext{
${ }^{1} \mathrm{~A}$ classic sociological treatment of social norms is Parson [17]. His views have since been critiziced for leaving little scope for individual choice, see Gouldner [9]. The rational choice school of sociologists recognizes the joint influence of social norms and economic incentives, see Coleman [6].

${ }^{2}$ Sources: Statistics Sweden (SCB) and National Social Insurance Board (RFV).

${ }^{3}$ For instance, in Sweden the corresponding figures in 1960 were 17 percent including the old-age pensioners and 8 percent excluding this group.
} 
normative pressure to earn one's income from work." Such a social norm may in part explain why far from all who are eligible for welfare programs participate in the programs. For example, this participation rate has been estimated to be only 40-70 percent in certain US welfare programs in the 1970's. Moffitt [16] finds econometric support from such data for a model of "welfare stigma," where the stigma is modeled as a fixed utility loss to recipients. ${ }^{4}$

However, it is possible that an increase in the number of people who receive welfare benefits may weaken the social norm to live off one's own work. Moreover, individuals who live off public transfers may over time come to value their leisure more. In an empirical study from the Netherlands, Engbersen et al [7] found that a majority of long term unemployed had stopped looking for work and that more than half of these had done so because they had found "other activities to give meaning to their lives: hobbies, voluntary work, or working in the informal economy."

While the existence of a social norm against living off other people's work is taken as given here, the intensity of the norm, as perceived by the individual, is endogenous in our model: it depends on the number of people adhering to it. More exactly we assume that living on transfers becomes relatively less embarrassing when more individuals do likewise. When the population fraction of transfer recipients is large (small), the individual's discomfort from such a life style is relatively weak (strong).

In our analysis of the interplay between economic incentives and this social norm we focus on two types of choice in connection with benefit systems - political and economic. First, the individual expresses her policy preferences as a voter, anticipating the consequences of the chosen policy for her own economic choice and for aggregate behavior - including the adherence in society at large to the social norm. Secondly, the individual maximizes her utility subject to given taxes, transfers, and the population fraction adhering to the norm.

We assume that every individual in her economic decision has a choice between two, and only two, alternatives: either to work full time or to live solely on public transfers. Many benefits in the real world indeed do require that the individual does not work; examples are unemployment benefits, sick benefits, early retirement, and disability pensions. However, individuals often do not have such a choice, and those who do need not have only two alternatives. Within limits, though, many individuals have some discretion to choose whether to utilize existing benefits or not, and they are more or less constrained to either work full time or not at all. The subsequent analysis can be extended to cases where only some individuals are entitled to benefits. ${ }^{5}$

\footnotetext{
${ }^{4}$ Moffitt could reject the hypothesis that the stigma depends on the size of the welfare transfer. He did not analyze the possibility that the stigma might depend on the participation rate itself.

${ }^{5}$ It is not difficult to generalize the present setting to one where there are three categories of individuals: those who are not entitled to transfers (group A), those who can choose between
} 
Whether the individual chooses to work or not depends on the after-tax wage, the level of public transfers, and the population fraction of transfer recipients. The tax rate and the per capita transfer are, in turn, determined in a political process. In the formal part of our analysis, every individual correctly foresees the share of transfer recipients resulting from any tax/transfer combination on the political agenda, and votes according to her preferences. We define political equilibrium in terms of an unbeatable policy (or Condorcet winner), i.e., a transfer/tax combination that balances the government budget and obtains a majority of votes against any alternative balanced tax/transfer combination.

We show that (generically) there exists at most one unbeatable policy. This policy is either a certain low-transfer policy that results in a minority of transfer recipients or a certain high-transfer policy that results in a majority of transfer recipients. In the basic version of the model, with no altruism and no risk for involuntary exclusion from work life, the former policy simply means no transfer and zero tax rate. When altruism and/or the risk of involuntary exclusion from work life is introduced this policy alternative is replaced by a certain positive transfer and tax rate. By way of computer calculations we show that monotonic changes in preferences can result in non-monotonic and even discontinuous changes in political equilibrium. In this sense, monotonic changes in exogenous factors may result in a "rise and fall" of the welfare state.

Theoretical and empirical research by economists on the effects of welfarestate benefit programs have, with the exception of the above-mentioned study by Moffitt [16], relied on purely economic incentives. Likewise, the extent of income redistribution has been examined in voting models where individual voting decisions are based on economic incentives, see Roberts [18] and Meltzer and Richard [15]. Our modeling of political equilibrium is similar to theirs. However, while the transfer in their model is granted to everyone, in our model it is granted only to those who don't work. Moreover, in Meltzer's and Richard's model individuals choose their hours of work on a continuous scale while in our model all individuals face a binary choice: full-time work or no work at all. A conceptually more fundamental difference is that social norms are absent from their models.

An early attempt to incorporate social norms in economic analysis is a study by Akerlof [1] on the role of social customs in a model of fair wages and unemployment. Our model of norms has similarities with that of Akerlof. However, Akerlof does not deal with welfare-state issues, nor are political decisions analyzed in his model.

From a technical viewpoint our analysis of social norms resembles models of interdependent preferences where aggregate behavior - such as average consump-

work and the transfer (group B), and those who have no choice but to live off the transfer (group C). The present analysis concerns the special case when all individuals belong to group $\mathrm{B}$. However, it applies readily to this more general setting, mutatis mutandis, granted the decisive voter belongs to group $\mathrm{B}$. This is the case if neihter group A nor group $\mathrm{C}$ contains a majority of the population. 
tion, excess demand, or average hours of work - enter the individual's utility function. See Basu [2], Becker [3], Blomquist [5], Granovetter [10], Granovetter and Soong [11], [12], and Schelling [19]. Social norms have also been analyzed in a recent paper by Bernheim [4] where adherence to social norms is obtained as an equilibrium outcome driven by individuals' wish to obtain social esteem. For an informal discussion of the interplay between economic incentives and social norms, see Lindbeck [13].

The paper is organized as follows: In section 2 we describe the individual's economic decision problem: whether to work or to apply for the transfer. In section 3 the government's budget constraint is introduced, and section 4 examines which balanced budget policies qualify as political equilibria under majority rule. In both sections we illustrate some comparative static properties of the model by way of computer calculations. Section 5 extends the model to encompass altruism, and section 6 generalizes the model to include an individual's risk of being excluded from work life. Section 7 concludes with a summary and a discussion of some directions for further research. All proofs are relegated to an appendix at the end of the paper.

\section{The model}

We assume a continuum of individuals with wages distributed according to some continuously differentiable cumulative probability distribution function $\Phi{ }^{6}$ There is a positive density $\varphi(w)=\Phi^{\prime}(w)$ at all positive wage levels $w$, and that no individual has a zero wage. Suppose also that the wage distribution $\Phi$ has a finite mean $\bar{w}$, and let its median be $\tilde{w}$. Let $\Phi^{-1}$ denote the inverse function to $\Phi$.

Each individual $i$ either works full time or does not work at all. In the first case, she consumes her after-tax wage earnings $(1-t) w_{i}$ and enjoys some leisure. We normalize this level of leisure to zero. Here $t$ is the tax rate on wage earnings and $w_{i}$ is her wage. In the second case, the individual receives a government transfer $T$. This transfer is exempted from taxation and is granted to anyone lacking other income. ${ }^{7}$ An individual who receives this transfer thus consumes $T$ and enjoys full-time leisure. Individuals may, however, also experience disutility from accepting the transfer due to embarrassment or social stigma associated with living on public transfers rather than on one's own work. Such embarrassment is likely to be weaker the more individuals in society live on the transfer. Thus, if the population share living on the transfer is $x$, and the disutility from accepting the transfer is $v(x)$, then $v$ may be taken to be a decreasing function. Phrased in terms of social norms: if the social norm is that the source of one's subsistence should be one's own work, then the intensity of discomfort when deviating from

\footnotetext{
${ }^{6}$ We take this distribution to be fixed and given, thus neglecting the possibility that taxes and transfers may (at least in the long run) influence factor incomes.

${ }^{7} \mathrm{~A}$ taxed transfer $T^{*}$ would be equivalent to a tax-free transfer $T=(1-t) T^{*}$.
} 
this norm is a decreasing function of the population share of deviators.

Each individual $i$ chooses to work if and only if that choice results in higher utility than living off the transfer. ${ }^{8}$ That is, she works if and only if

$$
u\left[(1-t) w_{i}\right]>u(T)+\mu-v(x)
$$

where $\mu \in \mathbb{R}$ is the utility difference between the leisure of living on the transfer and the intrinsic utility that one may derive from work life. ${ }^{9}$

We assume that the utility from consumption is a strictly increasing and concave function running from minus infinity at zero consumption to plus infinity at infinite consumption, and that the disutility of deviating from the norm is nonincreasing in the fraction of deviators:

(A1) $u: \mathbb{R}_{++} \rightarrow \mathbb{R}$ is twice continuously differentiable, with $u^{\prime}>0$, $u^{\prime \prime}<0, \lim _{c \rightarrow 0} u(c)=-\infty$, and $\lim _{c \rightarrow \infty} u(c)=+\infty$

(A2) $v:[0,1] \rightarrow \mathbb{R}$ is continuously differentiable, with $v^{\prime} \leq 0$.

Individuals take the tax rate $t$, transfer $T$, and population share $x$ of transfer recipients as exogenously given when deciding whether to work or to live on the transfer. For every combination of these three parameters, such that $0 \leq t<1$, $T>0$, and $0 \leq x \leq 1$, there exists a unique critical wage rate such that all individuals with lower wages choose not to work and those with higher wages choose to work. The critical wage, $w^{*}(t, T, x)$, is the unique solution to the equation

$$
u[(1-t) w]=u(T)+\mu-v(x) .
$$

Taking the inverse of the sub-utility function $u$ for consumption one sees that the critical wage is strictly increasing in the tax rate $t$ and transfer $T$, and that it is continuous and non-decreasing in the population share $x$ of transfer recipients:

$$
w^{*}(t, T, x)=\frac{1}{1-t} u^{-1}[u(T)+\mu-v(x)] .
$$

Having found the critical wage rate that separates workers from transfer recipients, we may identify the population share $x$ of transfer recipients with the population share of individuals with wages below this critical level:

\footnotetext{
${ }^{8}$ Only continuous income distributions will be considered so indifferent individuals can be ignored.

${ }^{9} \mathrm{Of}$ course the utility of leisure may depend on aggregate leisure in society - leisure may have a positive or negative social externality. However, for the sake of analytical clarity we neglect this and assume that the utility of leisure is independent of $x$. (In the present model aggregate leisure is montonically related to $x$, the share of transfer recipients.) Note, however, that the sum of the two terms $\mu$ and $v(x)$ may together represent the compound effect of social norm adherence and such an externality - if their joint effect is not negative.
} 

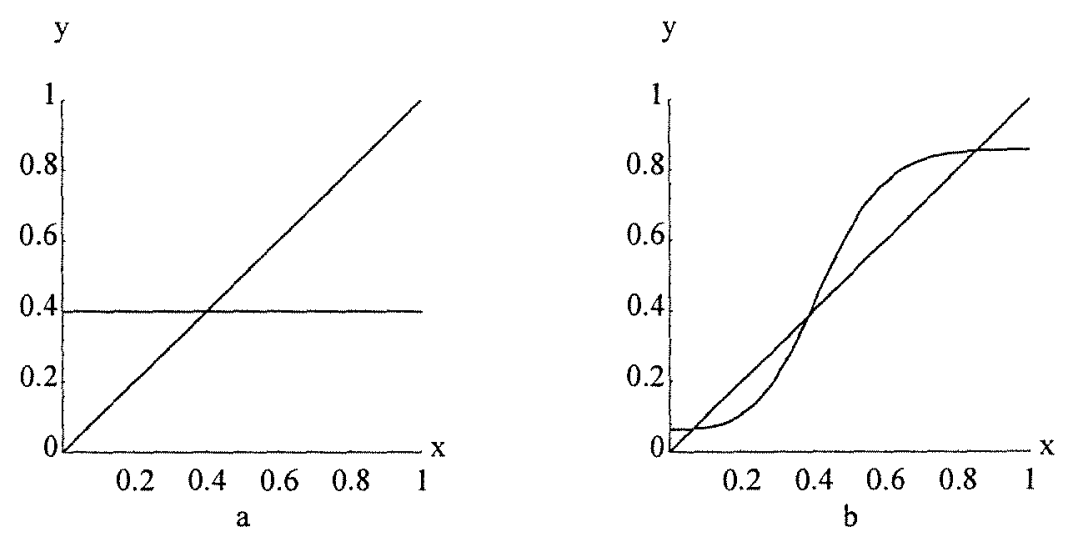

Figure 1: The fixed-point equation (4), under (a) "non-social" and (b) "social" preferences, respectively.

$$
x=\Phi\left[w^{*}(t, T, x)\right] .
$$

Conceptually, this is an equilibrium condition: If all individuals expect a population share of transfer recipients $x$, where $x$ satisfies this equation, then they will, in aggregate, make such individual choices that this population share will be realized. Mathematically, (4) is a fixed-point equation in $x$, with exogenous parameters $t$ and $T$. The right-hand side of the equation is a continuous function of $x$, mapping the unit interval $[0,1]$ into itself. Hence, there exists at least one population share $x^{*}$ satisfying equation (4), for any given tax rate $t<1$ and transfer $T>0$. Whether there exists more than one such population share (for a given tax rate and transfer) depends on the sub-utility functions $u$ and $v$ as well as on the wage distribution $\Phi$. A solution $x^{*}$ to (4) will be called an equilibrium population share.

In the special case when the disutility from deviating from the norm is independent of the population share $x$ of transfer recipients, then the equilibrium population share $x^{*}$ is unique. Inserting $v(x) \equiv \nu$ into equations $(2,4)$ we obtain

$$
x^{*}=\Phi\left(\frac{1}{1-t} u^{-1}[u(T)+\mu-\nu]\right) \text {. }
$$

This equation is illustrated in Figure 1 (a). The diagonal represents $y=x$, the left-hand side of the equation, while the horizontal line represents $y=$ $\Phi\left(\frac{1}{1-t} u^{-1}[u(T)+\mu-\nu]\right)$, its right-hand side.

The curve in Figure 1 (b) replaces the horizontal line in Figure 1 (a) and represents the right-hand side of equation (4) in a case when the disutility $v(x)$ 
decreases rapidly from a high to a low value at an intermediate value of $x$. The equation for this curve is $y=\Phi\left(\frac{1}{1-t} u^{-1}[u(T)+\mu-v(x)]\right)$. As shown in that diagram, equation (4) then has three solutions. The intuition for this multiplicity is that if the population share of transfer recipients is low (high) then the disutility from living on the transfer is high (low) and hence few (many) individuals do the same. ${ }^{10}$

Each equilibrium population share $x$ gives rise to a particular disutility $v(x)$ associated with deviations from the norm to work. Thus, economies with the same tax rates and transfers, factor incomes and preferences may differ in terms of the share of transfer recipients and the social stigma associated with this. In this respect the strength of the social norm, in comparison with the economic incentives, is endogenous.

These observations raise a number of policy questions. In particular, one may ask how the set of equilibrium population shares depends on the two policy instruments, the tax rate $t$ and transfer $T$. The following section considers this question in some detail.

\section{Balanced-budget equilibria}

The subsequent analysis requires some more notation and terminology. First, by a macro state we mean a triplet $s=(t, T, x)$ such that $t$ is a non-negative tax rate not exceeding one, $T$ is a non-negative transfer, and $x$ is a population share satisfying equation (4). Without loss of generality we exclude the case when all income is taxed and no transfer is given. For every tax/transfer pair there then exists at least one population share $x$ such that the associated triplet $s=(t, T, x)$ constitutes a macro state. We call the tax/transfer pair $(t, T)$ in such a triplet a policy, and write $p=(t, T)$.

So far, no connection has been assumed between the tax rate and the transfer. We restrict the subsequent analysis to those policies $p=(t, T)$ that balance the government budget. In view of the possibility that there may correspond multiple macro states $s=(t, T, x)$ to a given policy $p=(t, T)$, we impose the requirement of budget balance directly on the macro state. This balance requirement simply equates public spending on the transfer with public revenues from the wage tax.

The aggregate income from all individuals who work can be conveniently expressed in terms of the truncated expected-value function $\Psi$ defined by

$$
\Psi(w)=\int_{w}^{\infty} w^{\prime} \varphi\left(w^{\prime}\right) d w^{\prime} .
$$

\footnotetext{
${ }^{10}$ The cause of the multiplicity of equilibrium population shares is logically similar to that observed in Basu's [2], Granovetter's and Soong's [11], [12], Becker's [3], and Blomqvist's [5] analyses of preferences which depend on aggregate behavior.
} 
Thus $\Psi(w)$ is the wage sum for individuals with wages above $w$, normalized to per capita units. Clearly $\Psi$ is continuously differentiable and decreasing from the positive mean value $\bar{w}$ of the full wage distribution $\Phi$ toward zero as $w$ increases from zero toward infinity. The tax base, normalized to per capita units, is simply the value of this function evaluated at the critical wage rate $w^{*}(s)$ : in any macro state $s=(t, T, x)$ all individuals with higher wages work and pay the income tax, and no individual with a lower wage works. Since the income tax here is proportional, at rate $t$, the aggregate (per capita) tax revenue is simply $t \Psi\left[w^{*}(s)\right]$.

Similarly, aggregate (per capita) government expenditure on transfer payments is the transfer times the population share of transfer recipients. Thus a macro state $s=(t, T, x)$ balances the government budget if and only if

$$
T x=t \Psi\left[\Phi^{-1}(x)\right] .
$$

Macro states $s$ that satisfy this equation will be called balanced. Clearly the "zero-tax zero-transfer" macro state $s^{o}=(0,0,0)$ is a balanced macro state. We call a policy $p=(t, T)$ balanced if there exists some population share $x$ of transfer recipients such that the triplet $s=(t, T, x)$ constitutes a balanced macro state.

It is easily verified that to any policy $p=(t, T)$ there corresponds at most one population share such that the corresponding macro state is balanced. To see this, first suppose $T>0$. Then the left-hand side (aggregate transfer payments) of the budget equation (7) is strictly increasing in the population share $x$ of transfer recipients, while the right-hand side (aggregate tax revenues) is non-increasing in the same variable. Thus the budget equation is met by at most one population share $x$ in this case. Second, suppose $T=0$. Then all individuals choose to work and hence $x=0$.

It follows that there to every balanced policy exists exactly one population share $x$ of transfer recipients such that the corresponding macro state is balanced. Let $x=\xi(p)$ be the population share corresponding to a balanced policy $p$. In fact, the function $\xi$ can be shown to be a one-to-one relationship. In other words, for every population share of transfer recipients $x$ below 1 there exists exactly one policy $p=(t, T)$ such that the corresponding macro state is balanced. Formally:

Proposition 1 There exists a pair of functions, $f, F:[0,1) \rightarrow \mathbb{R}$, such that $t=f(x)$ and $T=F(x)$ uniquely determine the balanced macro state, $s=(t, T, x)$, associated with each population share $x \in[0,1)$ of transfer recipients. ${ }^{11}$

The tax function $f$ is implicitly defined by the identity $H[x, f(x)] \equiv 0$, where

$$
H(x, t)=u\left[(1-t) \Phi^{-1}(x)\right]-u\left[\frac{t}{x} \Psi\left[\Phi^{-1}(x)\right]\right]-\mu+v(x) .
$$

The function value $H(x, t)$ is the utility difference between the two choice alternatives - to work or not to work - for "the critical individual" in a balanced macro

\footnotetext{
${ }^{11}$ See Lindbeck, Nyberg and Weibull [14] for a proof.
} 

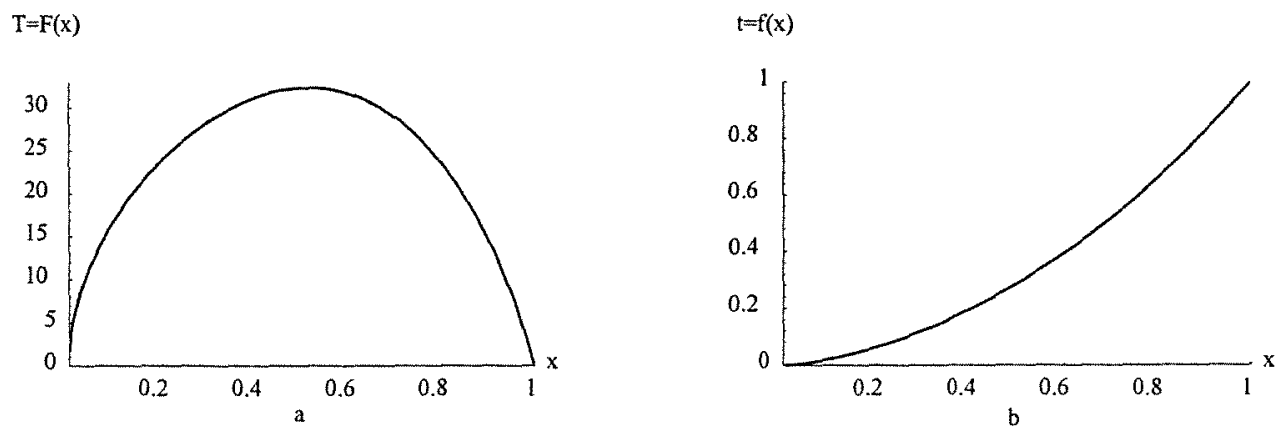

Figure 2: A transfer function $F$ with associated tax function $f$.

state where $0<x<1$. By "the critical individual" we mean an individual who earns the "critical" wage $w^{*}(s)$. By definition, the critical individual is indifferent between working and living on the transfer. Hence, in a balanced macro state it is necessary that $H(x, t)=0$.

To see the suggested interpretation of $H(x, t)$, first note that $\Phi^{-1}(x)$ equals the critical wage $w^{*}(s)$, an equality that follows directly from equation (4). Hence, $(1-t) \Phi^{-1}(x)$ is the critical individual's disposable income if she chooses to work. Accordingly, the first term in the expression for $H(x, t)$ is the utility resulting from this choice. Moreover, the budget balance requirement ( 7 ) forces the associated transfer to equal $t \Psi\left[\Phi^{-1}(x)\right] / x$. Thus the second term in the expression for $H(x, t)$ is (minus) the sub-utility of consuming that transfer. The third term is (minus) the utility from enjoying full time leisure, and the fourth term is the disutility from living off others' work. Hence, the second, third and fourth terms together represent the utility associated with the choice to live on the transfer.

Once the tax function $f$ has been identified, the transfer function $F$ is obtained directly from the government budget constraint: $F(x)=f(x) \Psi\left[\Phi^{-1}(x)\right] / x$. It is easily verified from the identity $H[x, f(x)] \equiv 0$ that as the population share increases toward 1 , so does the tax rate $t=f(x)$.

In a model based on purely economic incentives one would expect the tax rate to be higher when the population share of transfer recipients is higher. Indeed, if individuals' preferences are "non-social," i.e., independent of aggregate behavior $x$, then $f$ can be shown to be increasing. See Figure 2 for an illustration of the functions $F$ and $f$ in this case. However, in the presence of social norms $f$ need not be monotonic. The reason is that if the embarrassment of living on the transfer decreases drastically for a small increase in the population share of transfer recipients, say from $x$ to $x+\varepsilon$, then transfers become much more attractive and thus $T$ must decrease in order for $x+\varepsilon$ to be an equilibrium population share. If the compensating reduction in $T$ is large enough, then budget 

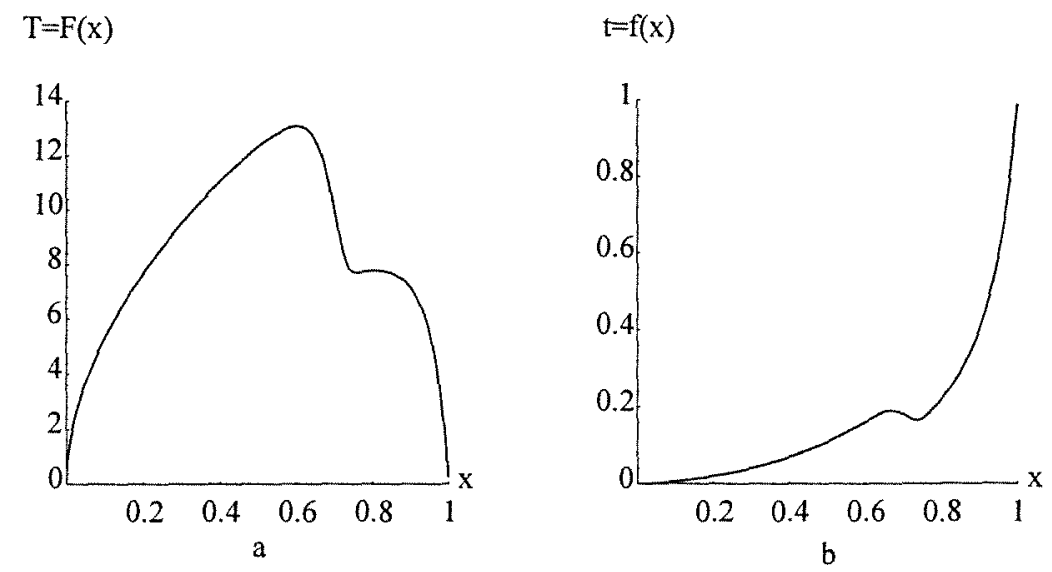

Figure 3: A non-concave transfer function $F$ and the associated non-monotonic $\operatorname{tax}$ function $f$.

balance requires the tax rate $t$ to decrease too (see Figure 3 for an illustration).

\subsection{Example}

The subsequent computer calculations are based on a logarithmic subutility function for consumption: $u(c)=\log (c)$ for all $c>0$. The identity $H[x, f(x)] \equiv 0$ then gives the following explicit expressions for the $\operatorname{tax}$ function $f$ :

$$
f(x)=\frac{x \Phi^{-1}(x)}{x \Phi^{-1}(x)+\Psi\left[\Phi^{-1}(x)\right] \exp [\mu-v(x)]}
$$

Insertion of this expression for the tax rate in the budget balance equation gives

$$
F(x)=\frac{\Phi^{-1}(x) \Psi\left[\Phi^{-1}(x)\right]}{x \Phi^{-1}(x)+\Psi\left[\Phi^{-1}(x)\right] \exp [\mu-v(x)]} .
$$

It follows from these expressions that a shift in the wage distribution such that all individuals' wages are multiplied by the same factor, $\lambda>0$, results in no change in the tax function $f$ and a proportional change in the transfer function $F$. This results from the observation that $\Phi^{-1}(x)$ and $\Psi\left[\Phi^{-1}(x)\right]$ then are replaced by $\lambda \Phi^{-1}(x)$ and $\lambda \Psi\left[\Phi^{-1}(x)\right]$, respectively. ${ }^{12}$

\footnotetext{
${ }^{12}$ The graphic illustrations in this section are based on Weibull distributed wages. The Weibull distribution is governed by three parameters. One determines the lower end of its support, another determines the scale along its support. These two parameters will be kept fixed throughout. The third, here denoted $c$, determines the concentration of the distribution. High values of $c$ correspond to a high degree of concentration (wage equality). More precisely,
} 

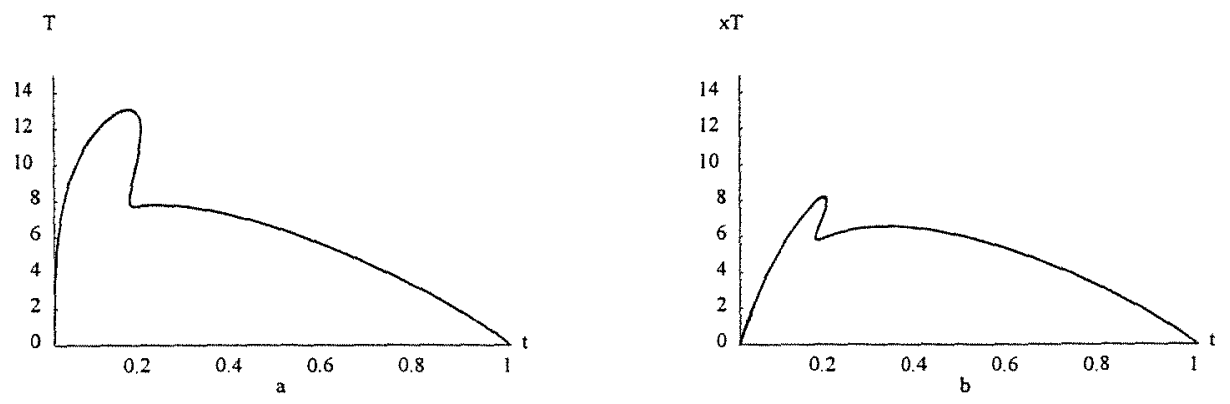

Figure 4: (a) The set of balanced policies, (b) the associated "Laffer curve."

We noted above that the multiplicity of solutions to the fixed-point equation (4) vanishes when one imposes the public budget constraint. For each tax/transfer pair there then exists at most one population share $x$ of transfer recipients. Nevertheless, there remains, at least a priori, the possibility that for certain tax rates $t$ there exists more than one pair $(T, x)$ that balances the public budget. Multiplicity of this type implies a certain relation between the associated transfer/recipient pairs: if both $(T, x)$ and $\left(T^{\prime}, x^{\prime}\right)$ are compatible with balancing the budget at the same tax rate $t$, and the transfer $T^{\prime}$ is higher than $T$, then the population share $x^{\prime}$ must be smaller than the population share $x$. Because otherwise public spending would be higher in $\left(T^{\prime}, x^{\prime}\right)$ while the tax base would be smaller (the tax rate is by hypothesis the same). Such multiplicity, at a given tax rate, clearly requires social preferences. If fewer individuals choose the higher transfer $T^{\prime}$ then the social norm against living on transfers must be stronger in $\left(T^{\prime}, x^{\prime}\right)$ than in $(T, x)$.

Figure 4 (a) illustrates that multiplicity of transfer/recipient pairs $(T, x)$ is a real possibility in our model for certain tax rates. The curve represents the set of balanced policies, based on the same numerical specification as Figure 3. Note the folding of the curve above an interval of tax rates near 20 percent. For each of these tax rates $t$ there are three values of $T$ such that the policy $p=(t, T)$ belongs to the curve (at the end of this interval there are two such values of $T)$. Let these three policies be denoted $p=(t, T), p^{\prime}=\left(t, T^{\prime}\right)$ and $p^{\prime \prime}=\left(t, T^{\prime \prime}\right)$, respectively, with $T<T^{\prime}<T^{\prime \prime}$. As argued above the corresponding unique population shares must satisfy $x>x^{\prime}>x^{\prime \prime}$. In sum: for such tax rates $t$ there exist three transfer/recipient pairs.

a random variable $X$ is Weibull $(a, b, c)$ distributed if for all $x \geq a$ :

$$
\operatorname{Pr}(X \leq x)=1-e^{-\left(\frac{x-a}{b}\right)^{c}} .
$$


It turns out that there are connections between the multiplicity of solutions to the fixed point equation (4), as illustrated in Figure 1 (b), and folds in the balanced-policy curve, shown in Figure 4. First, policies $p$ on upward sloping segments of this curve correspond to fixed points in equation (4) where the curve in Figure 1 (b) intersects the $45^{\circ}$-line from above (the lowest and highest fixed point). Second, policies on the backward running part of the fold correspond to fixed points where the curve in Figure 1 (b) intersects the $45^{\circ}$-line from below (the middle fixed point). Third, all points on the initial upward sloping segment of the balanced-policy curve in Figure 4, starting at the zero-tax policy $p^{\circ}=(0,0)$, correspond to the lowest fixed point in equation (4) - the smallest population share of transfer recipients compatible with the given policy $p$ on that segment of the curve.

Before discussing implications for dynamic stability of macro states we need to define a dynamic. Indeed, there is a natural class of dynamics in the expectation formation behind the fixed-point equation (4). Suppose a policy $p$ has been chosen and that all individuals expect some accompanying fraction $x^{e}$ of transfer recipients when they themselves decide whether or not to work. The resulting, true, population share of transfer recipients then is $x=\Phi\left[w^{*}\left(t, T, x^{e}\right)\right]$. If $x$ equals $x^{e}$, then the aggregate of individuals have succeeded in finding a solution to the fixed-point equation (4) ("rational expectations"). However, suppose $x<x^{e}$, i.e., fewer individuals than expected opted for the transfer. It is then plausible that some individuals who opted for the transfer will now opt for work (the embarrassment was greater than expected). Likewise, if $x>x^{e}$ some individuals who opted for work will now shift to the transfer. In such an adaptation process $x^{e}$ would increase (stay put, decrease) if $\Phi\left[w^{*}\left(t, T, x^{e}\right)\right]$ exceeds (equals, falls short of) $x^{e}$. Geometrically, $x^{e}$ will increase (decrease) where the curve in Figure 1 (b) lies above (below) the $45^{\circ}$-line. The claims above imply that fixed points where the curve intersects the $45^{\circ}$-line from above are stable (unstable) in such a dynamics. In particular, policies $p$ on the backward-running segment of the fold in the balanced-policy curve in Figure 4 correspond to unstable fixed points $x$. Hence, it seems implausible that such policies can in practice be maintained.

It remains to prove the three claims made above. For this purpose, first note that, as we move along the balanced-policy curve from the zero-tax policy $p^{0}=(0,0)$ toward the unit-tax policy $p^{1}=(1,0)$, the population share $x$ of transfer recipients increases monotonically from zero to one. ${ }^{13}$ This follows from the continuity of the functions $f$ and $F$, that together map population shares $x$ to policies $p$, in combination with the fact that to each $x \in[0,1)$ there corresponds exactly one point $p$ on the curve (one balanced policy). Next, since the critical wage $w^{*}(t, T, x)$ is increasing in $t$ and in $T$ (for each $x$ ), the curve in Figure 1 (b) shifts up (down) if both $t$ and $T$ increase (decrease). This implies that segments

\footnotetext{
${ }^{13}$ This implies the earlier observation concerning the fold in the balanced-policy curve that $x>x^{\prime}>x^{\prime \prime}$.
} 
of the balanced-policy curve in Figure 4 where both $t$ and $T$ increase correspond to fixed points $x$ where the curve in Figure 1 (b) intersect the $45^{\circ}$-line from above (below). This proves the first two claims. The third claim follows from the first claim in conjunction with the observation that when $p^{\circ}=(0,0)$ the unique fixed point is $x=0$. Hence, by continuity, policies $p$ on the initial segment of the balanced-policy curve correspond to the lowest fixed point (if multiple fixed points exist) that is compatible with $p$.

Multiplication of a transfer payment $T$ with an associated population share $x$ of transfer recipients determines total government expenditures (per capita), $x T$. See Figure 4 (b) for a plot of $x T$ against the tax rate $t$; a "Laffer curve." Note that the fold in diagram (a) is carried over to this new curve.

\section{Political equilibrium}

The purest political equilibrium notion for this model seems to be that of an unbeatable policy (or Condorcet winner), by which we mean a balanced policy $p$ such that no other balanced policy is preferred by a majority of the population.

In order to render this notion more precise, we first define individual preferences over policies. The utility to individual $i$ under a balanced policy $p=(t, T)$ is simply the highest of two utility levels, one for each of the two economic choice alternatives that are available to the individual:

$$
U_{i}(p)=\max \left\{u\left[(1-t) w_{i}\right], u(T)+\mu-v(x)\right\},
$$

where $x=\xi(p)$. Using this fact, we say that individual $i$ prefers policy $p^{\prime}$ to policy $p$ if $U_{i}(p)<U_{i}\left(p^{\prime}\right)$. Accordingly, policy $p^{\prime}$ wins over policy $p$ in a majority vote if there are more individuals who prefer $p^{\prime}$ to $p$ than there are individuals who prefer $p$ to $p^{\prime}$. A policy $p$ is unbeatable if no policy $p^{\prime}$ wins over $p$. Note that by this definition indifferent voters, i.e., voters $i$ such that $U_{i}(p)=U_{i}\left(p^{\prime}\right)$, are split equal between the two policies.

It is evident that in this basic version of the model, without altruism and without any risk of being involuntarily excluded from work life, no policy resulting in a positive but small share of transfer recipients is unbeatable; such a policy would lose against the "zero transfer" policy $p^{o}=(0,0)$. Individuals who work in the proposed balanced macro state are better off under $p^{\circ}$, and they constitute a majority. Consequently, an unbeatable policy is either the zero policy $p^{o}$ or a policy that has a positive tax rate and transfer such that a majority of individuals choose not to work in equilibrium.

It turns out that there is only one alternative to the zero-tax policy $p^{o}$, viz. the policy $\hat{p}$ that is optimal from the viewpoint of transfer recipients and (in case there is more than one optimal policy for transfer recipients) that requires the lowest tax rate among such optimal policies. Any other such policy can be beaten by the tax-rate minimizing policy since transfer recipients are indifferent 
between the two policies and workers-cum-taxpayers prefer the policy with the lower tax-rate. We proceed by first defining this alternative policy $\hat{p}$ and then state the results and provide some intuition for them.

The range of policies that are optimal from the viewpoint of transfer recipients are those that result in population shares $x$ of transfer recipients that belong to the set

$$
\hat{X}=\arg \max _{x \in(0,1)}(u[F(x)]+\mu-v(x)) .
$$

Let $\hat{x}$ be the smallest population share in this set: $\hat{x}=\min \hat{X}$. It can be shown that this population share results in the lowest tax rate among all population shares $x$ in $\hat{X} .{ }^{14}$ Let $\hat{p}=(f(\hat{x}), F(\hat{x}))$ be the associated policy.

On the basis of this observation one can show that the policy $\hat{p}$ is the only alternative political equilibrium to the zero-tax policy $p^{o}$ :

Proposition 2 If $p=(t, T)$ is unbeatable and $t>0$, then $p=\hat{p}$, where $\hat{x}=$ $\xi(\hat{p}) \geq \frac{1}{2}$.

(See Appendix for a proof.)

In particular, if the wage distribution and preferences are such that the population share $\hat{x}$ is less than one half, then the only potential political equilibrium is the zero-tax zero-transfer policy.

Proposition 2 leads to the question under what conditions the zero-tax policy $p^{\circ}$ and/or the positive-tax policy $\hat{p}$ is unbeatable. The following two results give precise answers to parts of this question. Let us define the highest feasible utility level for transfer recipients in any balanced macro state in which they constitute a (weak) majority:

$$
\beta=\max _{\frac{1}{2} \leq x<1}(u[F(x)]+\mu-v(x)) .
$$

A necessary and sufficient condition for the zero-tax policy $p^{o}$ to be unbeatable is simply that this utility level does not exceed the utility of the median wage earner from his or her untaxed wage, $u(\tilde{w})$ :

Proposition 3 The zero-tax policy $p^{o}$ is unbeatable if and only if $u(\tilde{w}) \geq \beta$. No other policy is unbeatable when $u(\tilde{w})>\beta$.

(See Appendix for a proof.)

Similarly, a necessary and sufficient condition for a positive-tax policy $\hat{p}$, such that a majority lives on transfers, to be unbeatable, is that the resulting utility for a transfer recipient is not lower than $u(\tilde{w})$ :

\footnotetext{
${ }^{14}$ The set $\hat{X}$ can be shown to be nonempty and compact. Thus it contains its minimal element. Moreover, for any $x, x^{\prime} \in \hat{X}, x<x^{\prime} \Rightarrow f(x)<f\left(x^{\prime}\right)$. See Lindbeck, Nyberg and Weibull [14] for a proof.
} 
Proposition 4 Suppose $\hat{x}>\frac{1}{2}$. Then the positive-tax policy $\hat{p}$ is unbeatable if and only if $u(\tilde{w}) \leq \beta$.

(See Appendix for a proof.)

Thus, political equilibrium depends on whether $\hat{x}$ falls short of or exceeds one half, and whether or not the utility $u(\tilde{w})$ to the median wage earner from her untaxed wage exceeds $\beta$. Generically, i.e., barring ties, the following three cases are the only possibilities: (A) $u(\tilde{w})>\beta$, (B) $u(\tilde{w})<\beta$ and $\hat{x}>\frac{1}{2}$, and (C) $u(\tilde{w})<\beta$ and $\hat{x}<\frac{1}{2}$. It follows from propositions $1-3$ that a unique political equilibrium exists in cases $(A)$ and $(B)$. In case $(A)$ the unique equilibrium is the zero-tax policy $p^{\circ}$. This follows immediately from proposition 3: The median wage earner prefers the zero-tax policy over the best transfer possible among those that result in a majority of transfer recipients $(u(\tilde{w})>\beta)$. In case (B) it is the positive tax policy $\hat{p}$ that is the unique political equilibrium, for two reasons. First, the median wage earner prefers the best transfer possible, among those that result in a majority of transfer recipients, to the zero-tax policy $(u(\tilde{w})<\beta)$. Hence, $p^{\circ}$ is not unbeatable (proposition 3). Secondly, the optimal policy from the viewpoint of transfer recipients, among all balanced-budget policies, results in a majority of transfer recipients. Thus $\hat{p}$ is unbeatable (proposition 4). No political equilibrium exists in case $(C)$. In that case there exists a transfer that results in a majority of transfer recipients and that the median wage earner prefers over working at her untaxed wage $(u(\tilde{w})<\beta)$. Hence such a policy beats the zero-tax policy $p^{o}$ (proposition 3 ). Moreover, the positive-tax policy, $\hat{p}$, the only remaining alternative for political equilibrium, results in a minority of transfer recipients $\left(\hat{x}<\frac{1}{2}\right)$ and is therefore not unbeatable (proposition 2 ).

\subsection{The value of leisure}

As indicated in the introduction, there is a possibility that transfer recipients learn over time to use and appreciate their leisure. Technically, this corresponds to an increase over time in the utility parameter $\mu$, for those who are transfer recipients. If such a parameter drift is restricted to that subpopulation, then the equilibrium outcome does not change. Those who decided to live on the transfer will only feel more satisfied with their choice, and those who decided to live off their own work will not experience any change in their preferences. However, it is plausible that such a preference drift among transfer recipients spreads to those who decided to work; they realize over time that the life as transfer recipients may be more attractive than they had previously thought. We here study the effects of such a preference drift in society as a whole by way of a numerical comparative statics exercise. Hence, the results can either be interpreted as a comparison of societies with differing tastes $\mu$ for leisure or as one society that experiences a sequence of unanticipated increases in $\mu$, increases that may for instance be due to such a social learning process as indicated above. 

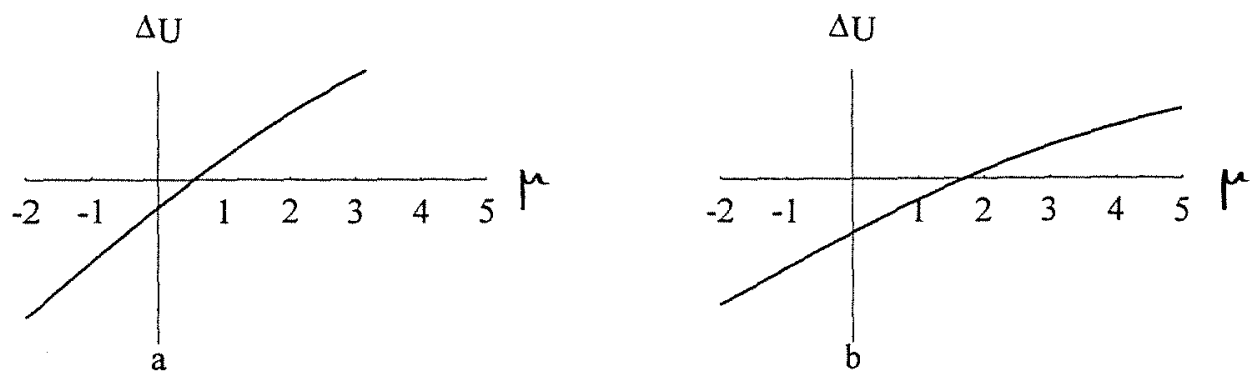

Figure 5: The difference in utility for the median wage earner between living off the transfer and off her untaxed wage, as a function of the utility of leisure, $\mu$.

In order to highlight the role of the taste $\mu$ for leisure (more exactly the utility difference between leisure as a transfer recipient and the intrinsic utility that one may derive from work life), this comparative statics exercise is carried out under the simplifying assumption that the strength of the social norm is kept constant. Hence, we here set $v(x) \equiv 0$. The set $\hat{X}$ then consists of those population shares $x$ at which the transfer $T=F(x)$ is maximal, and the point $\hat{x}$ is its smallest element. It turns out that the higher the value of leisure $\mu$ the larger is the population share of transfer recipients at which the transfer is maximal. This suggests that a positive tax policy is more likely to be unbeatable in the case of a more unequal wage distribution, in the sense that $\hat{x}$ then exceeds $\frac{1}{2}$ for a wider range of $\mu$-values (see proposition 2). This observation conforms qualitatively with Meltzer's and Richard's [15] result that a large difference between the mean-value and median of the wage distribution results in large redistributions.

This is supported by the graphs in Figure 5. According to proposition 4 the policy supporting $\hat{x}$ is unbeatable if the median wage earner rather lives on the maximal transfer $\hat{T}=F(\hat{x})$ than works under the zero-tax policy. Figure 5 illustrates the utility difference, $\Delta U$, between these alternatives for the median wage earner as a function of $\mu$. Panel (a) is based on a more dispersed wage distribution than panel (b). Not surprisingly, both graphs show that transfers become more attractive as the value of leisure, $\mu$, increases. Indeed, for a sufficiently high valuation of leisure a certain positive-tax policy becomes unbeatable, i.e., the median wage earner prefers to live on the transfer. The critical value of $\mu$, below which the zero-tax policy, $p^{\circ}$, is unbeatable, depends on the wage distribution. In Figure 5 the critical values are approximately 0.6 and 1.7.

The effect of an increasing valuation of leisure on political equilibrium is illustrated in Figure 6 using the same wage distribution as in panel (b) in Figure 5. For $\mu$ below the critical value, 1.7, the zero-tax policy is unbeatable. Above the critical value, however, $\mu$ determines the properties of the unbeatable policy. 

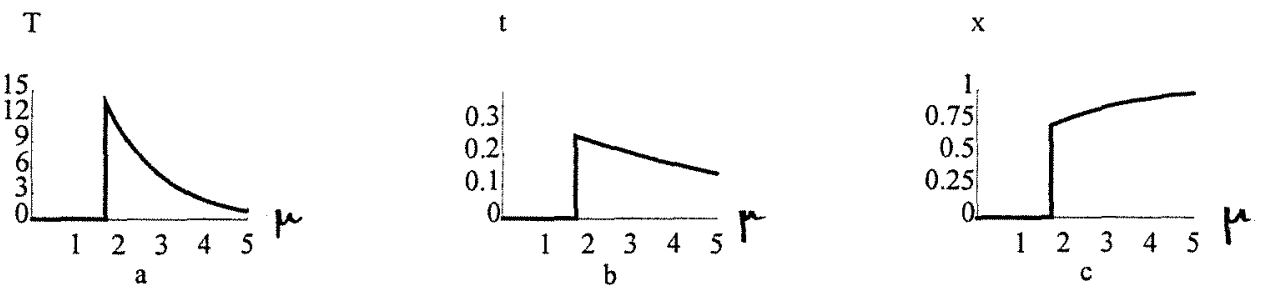

Figure 6: The unbeatable policy, and the associated population share $x$, as functions of the utility of leisure, $\mu$.

As may be expected, a higher valuation of leisure increases the political support, $\hat{x}$, for a positive tax policy (panel (c)). Moreover, an increase in $\mu$ simultaneously leads to lower per capita transfers (panel (a)). This is accompanied by a decreasing tax rate (panel (b)). Thus, starting from a situation where the zero-tax policy is unbeatable, a gradual increase in the valuation of leisure will eventually induce a sudden shift in the political equilibrium outcome to a highly redistributive policy. Further increases in $\mu$ result in a lower transfer, albeit to an increasing fraction of the population. ${ }^{15}$

\section{Altruism}

The above model is half-hearted concerning the social nature of preferences. It does allow for preferences to be social in the sense that an individual's private economic decision may be influenced by the choices of others. However, an individual's political voting decision is assumed to be independent of the policy consequences for other individuals in society. Presumably most individuals have preferences that are social also in this respect. Here we provide an extension of the model in this direction.

We focus on "Rawlsian altruism," i.e., an altruistic concern for those who are worst off in society. Since all individuals have the same preferences over their own consumption and leisure, the minimal private subutility, across all individuals in a macro state with a positive share of transfer recipients, is $u(T)+\mu-v(x)$. We now add the assumption that all individuals in society are equally altruistic,

\footnotetext{
${ }^{15}$ The above mentioned two effects do not hinge on the logarithmic form of the subutility function for consumption: for non-social preferences they hold for any function $u$ that satisfies condition (A1). From the identity $H[x, f(x)] \equiv 0$ it is easy to see that the maximal transfer decreases in $\mu$. Consider the effect of an increase in $\mu$ for any given $x$. Then $t$ must decrease, which, for a given $x$, means that $T$ decreases in the same proportion. Since this holds for all $x$ between zero and one, both functions $F$ and $f$ shift point-wise down in $\mu$. Consequently, the maximal $T$-value, $\hat{T}$, must decrease. To show that the corresponding population share (or political support) $\hat{x}$ is non-decreasing in $\mu$ is straightforward but somewhat tedious.
} 
and that they have additively separable utility functions that combine "private" utility from own consumption, leisure, and source of subsistence, with "altruistic" utility from others' welfare.

Letting a non-negative weight $\alpha$ be attached to the altruistic component, we obtain the following extension of the model developed in the previous sections. ${ }^{16}$ Since the altruistic term is the same, irrespective of whether an individual chooses to work or live off the transfer, altruism has no effect on an individual's economic decisions: all individuals with wages above (below) the critical wage $w^{*}(t, x, T)$ still choose to work (live on the transfer).

However, altruism does potentially affect individuals' political behavior. Consider again individual $i$ with wage $w_{i}$, now faced with a voting decision between two (balanced-budget) policies $p$ and $p^{\prime}$. Let the induced macro states be $s$ and $s^{\prime}$. In section 4 , we defined $U_{i}(p)$ and $U_{i}\left(p^{\prime}\right)$ as the individual's "private" utility from these two alternatives. Let $\bar{U}_{i}(p)$ and $\bar{U}_{i}\left(p^{\prime}\right)$ be her total (private and altruistic) utility from the alternatives. Then

$$
\bar{U}_{i}(p)=U_{i}(p)+\alpha[u(T)+\mu-v(x)]
$$

and likewise for $\bar{U}_{i}\left(p^{\prime}\right)$, granted that both $x$ and $x^{\prime}$ are positive.

The special case of assigning zero weight to altruism corresponds to the original model. If individuals instead are altruistic, i.e., if $\alpha$ is positive, then, unlike in the original model, the zero-tax policy can never be unbeatable. This follows from our assumption (A1) that the utility from zero consumption is minus infinity, along with the assumption that there are individuals with (virtually) zero wage. Hence, an altruist prefers to give some transfer to these.

So what policy will now play the role of the zero-tax policy $p^{o}$ ? It turns out that workers (in a given macro state) with different wages will in general prefer different transfer levels. Hence, no single policy plays the role of $p^{o}$. However, if the utility from consumption is logarithmic then all those who work have the same preferences over transfers. In order to substantiate these claims, first note that the (total) utility to a working individual $i$ in some macro state $s=(t, T, x)$ is $W_{i}(x)$, where $t=f(x), T=F(x)$, and

$$
W_{i}(x)=u\left([1-f(x)] w_{i}\right)+\alpha(u[F(x)]+\mu-v(x)) .
$$

We see from this definition that $W_{i}(x) \rightarrow-\infty$ as $x \rightarrow 0$ and as $x \rightarrow 1$, and thus all workers in any macro state prefer shares of transfer recipients that lie strictly between zero and one. Consequently, $T=F(x)>0$ in any unbeatable policy.

A necessary condition for some interior population share $x$ to maximize $W_{i}(x)$ is the first-order condition $W_{i}^{\prime}(x)=0$, which is equivalent to

\footnotetext{
${ }^{16}$ One may allow for the possibility that the weight attached to the worst off individuals may depend on the distribution $\Phi$ of gross incomes; in which case $\alpha$ is replaced by $\alpha(\Phi)$.
} 


$$
u^{\prime}\left([1-f(x)] w_{i}\right) w_{i} f^{\prime}(x)=\alpha\left[u^{\prime}(F(x)) F^{\prime}(x)-v^{\prime}(x)\right]
$$

In general, the solution (set) to this equation depends on $w_{i}$, the individual's wage. However, if the utility from consumption is logarithmic, $u(c) \equiv \log (c)$, then all $w_{i}$ cancel and all workers prefer the same $x>0$. Furthermore, suppose that the above first-order condition has a unique solution $x^{+}$, and let $p^{+}$be the associated policy, i.e., $p^{+}=\left(t^{+}, T^{+}\right)$, where $t^{+}=f\left(x^{+}\right)$and $T^{+}=F\left(x^{+}\right)$are both positive.

Now the logic of section 4 kicks in. The set $\hat{X}$ is still the ideal set for transfer recipients - their utility has only been multiplied by the constant and positive factor $1+\alpha$. Hence, $\hat{p}$ is still a potentially unbeatable policy. Proposition 2 is modified only in that the condition " $t>0$ " is replaced by " $t>t^{+}$" and propositions 3 and 4 remain intact if $\beta$ is replaced by

$$
\bar{\beta}=[1+\alpha] \beta,
$$

and $u(\tilde{w})$ is replaced by

$$
\bar{u}(\tilde{w})=u\left[\left(1-t^{+}\right) \tilde{w}\right]+\alpha\left(u\left(T^{+}\right)+\mu-v\left(x^{+}\right)\right) .
$$

The qualitative features of the analysis of political equilibrium in section 4 thus remain intact. The only difference is that the zero-tax policy $p^{o}$ is replaced by a positive-tax policy $p^{+}$. In other words, the political equilibrium is either the "high-tax" policy $\hat{p}$ supported by a majority of transfer recipients, or the "lowtax" policy $p^{+}$supported by a majority of workers who tax themselves because of altruistic concerns for a minority of transfer recipients.

\section{Involuntary exclusion from work}

In practice many individuals who would prefer to work are excluded from work life. This may be due to unemployment, illness etc. We now proceed to include this possibility in the model. Suppose some fraction $\lambda$, where $0 \leq \lambda<1$, do not have a choice whether or not to work; they are forced by external circumstances ("bad luck") to live off the transfer. For the sake of analytical simplicity we here take this fraction to be the same for all wage levels, and we presume that the fraction is known by all individuals.

If $x$ is the total population share of transfer recipients, voluntary and forced, then the fixed-point equation (4) that determines $x$ is generalized to

$$
x=\lambda+(1-\lambda) \Phi\left[w^{*}(t, T, x)\right],
$$

with the critical wage $w^{*}(t, T, x)$ defined as before. Here $v(x)$ represents the embarrassment of deviating from the social norm to live off one's own work, 
given the population share $x$ of transfer recipients. It could be argued that this embarrassment should be a function of the share $x-\lambda$ of individuals who voluntarily choose to deviate from the norm. However, one may define $v$ by setting $v(x)=\tilde{v}(x-\lambda)$ for all $x \geq \lambda$, where $\tilde{v}(y)$, for any $y \in[0,1-\lambda)$, is the embarrassment of deviating from the social norm when the population share of voluntary deviators is $y$. Hence, without any loss of generality with regard to the basis for the embarrassment we may still take $v$ to be as in assumption (A2).

Clearly, any equilibrating population share $x$ in equation (19) will be a number $x \in[\lambda, 1)$. This equation has at least one solution, for the same reason as in the case of (4). Thus, the discussion and analysis in section 2 remains intact once (4) has been replaced by (19). This is also true for section 3, dealing with the governmental budget balance, mutatis mutandis. The change is that the tax base is now reduced by the factor $1-\lambda$, so the budget equation (7) is generalized to

$$
T x=(1-\lambda) t \Psi\left[w^{*}(s)\right] .
$$

Accordingly, the domain of the tax and transfer functions, $f$ and $F$, is now restricted to the interval $[\lambda, 1)$, the argument in the second term in equation (8) is multiplied with the (positive) factor $1-\lambda$, and so is the expression for the transfer $F(x)$, given in the text.

As for political equilibrium, analyzed in section 4 , the function $\xi$, mapping balanced policies to population shares, is a one-to-one mapping between balanced policies and population shares in the interval $[\lambda, 1)$. Moreover, the range of possible cases is spanned between two polar cases. In the first case all individuals know before they vote whether they are forced to live on the transfer. In this case, that population share, which has size $\lambda$, will vote for the policy that is best for transfer recipients (irrespective of whether these voters are altruistic or not). The other voters will have policy preferences just as in the basic model version, given by equation (11).

In the opposite polar case, on which we focus here, no individual knows before she votes whether she will be forced to live on the transfer. Under the maintained hypothesis that all individuals face the same probability $\lambda$ of being so forced, and presuming that the utility functions are von Neumann-Morgenstern utility functions, the expected utility from a policy $p$ to an individual with wage $w_{i}$ is

$$
\begin{aligned}
V_{i}(p) & =\lambda[u(T)+\mu-v(x)]+(1-\lambda) U_{i}(p) \\
& =(1-\lambda)\left(U_{i}(p)+\frac{\lambda}{1-\lambda}[u(T)+\mu-v(x)]\right),
\end{aligned}
$$

where $U_{i}(p)$ is the utility to a worker, see definition in equation (11). In other words, individuals have the same policy preferences as if they were Rawlsian altruists with the weight $\alpha=\frac{\lambda}{1-\lambda}$ given to the welfare of the worst off individual in society, see section 5 . 
Hence, the formal analysis of political equilibrium is identical to that in section 5. Indeed, these two elements can be combined. If all individuals face the same (post voting) risk $\lambda$ of being forced to live off the transfer, and all individuals are equally altruistic, with weight $\alpha$, then their economic decisions will be made as indicated in the present section, and their political behavior will be as indicated in section 5 , with weight $\frac{\alpha+\lambda}{1-\lambda}$.

\section{Conclusions and directions for further research}

When social norms are introduced in a microeconomic model one might fear a plethora of possible outcomes - that "anything can happen." This fear turns out to be unjustified in the present setting. The range of possible outcomes is in fact highly restricted. Essentially, there are only two alternatives: a low-tax society with a minority of transfer recipients or a high-tax society with a majority of transfer recipients. Which of these two potential equilibria will materialize depends on preferences and on the wage distribution.

If the disutility from deviating from the social norm is highly sensitive to the fraction of deviators and the impact of the norm is important to the individual in comparison with her preference for consumption and leisure, then certain tax rates are consistent with multiple combinations of per capita transfer levels and fractions of benefit recipients. These combinations imply fulfilled expectations, a balanced budget but not necessarily political equilibrium. In a high per-capita transfer combination (with a small share of transfer recipients) the disutility of living from others work is high, while in a low transfer combination (with a large share of transfer recipients) this disutility is low.

The assumption in the model of perfect foresight in individuals' economic and political choice may be unrealistic, perhaps especially in their political choice. The reason is that the latter concerns a whole menu of alternative and yet unrealized tax rates and transfer levels with accompanying population shares of transfer recipients. It is tempting to speculate that individuals' perception of changes in the number of beneficiaries is subject to some inertia when changes in the tax rate and/or transfer level are considered by the electorate.

One may hypothesize that voters, when faced with a policy proposal, underestimate the resulting change in the population share of transfer recipients. Citizens in a low-transfer society may therefore vote for more generous programs than if they had correctly anticipated the long term socio-economic adjustment. If the benefit systems become more generous, more individuals than expected may choose to live on benefits because others do. In this sense the welfare state may overshoot. As a consequence a budget deficit may emerge. Restoring budget balance may require increased tax rates and/or reduced transfer levels. If the first path - increased tax rates - is followed it would become even more attractive to live on transfers. This may generate a "vicious circle" of more and more benefi- 
ciaries and yet higher tax rates. If instead the (per capita) transfer levels are cut, then transfer recipients will have to accept lower transfers and thus some transfer recipients will choose to work instead. This will strengthen the social norm for work and this will induce more transfer recipients to switch to work life. In this case a budget surplus may result. In sum: the endogeneity of the strength of the social norm for work seems to destabilize the budget balance process.

Consider another thought experiment, regarding the case where a tax rate $t$ is consistent with more than one combination $(T, x)$ of transfer level and population share of transfer recipients. Suppose the government gradually increases the tax rate $t$ and correctly adjusts the transfer $T$ so as to maintain budget balance, starting from a point to the left of the fold in Figure 4 (a). If expectations concerning the population share $x$ exhibit inertia then the policy $p=(t, T)$ continues to slide smoothly along the fold, until it turns vertically down. There, a marginal increase of $t$ results in a finite drop in $T$ and a switch by a sizeable population share from working to living on transfers. Further increases of $t$ lead to gradual reductions of the transfer. The switching individuals accept a lower transfer because the increased share of transfer recipients reduces the disutility associated with not working. A gradual policy shift has resulted in a shock to the social value attached to work. Reversing the thought experiment, gradually reducing the tax rate from a point above the fold interval, results in a policy $p$ that slides along the lower side of the fold, and then jumps up accompanied by a sudden fall in the share of transfer recipients. Note that this jump takes place at a lower tax rate than the downward jump described before.

The present analysis suggests several avenues for future research. First, it might be valuable to formalize the heuristic discussion above about inertia. Second, an obvious modification of our model is to allow for marginal adjustments in individual hours of work. ${ }^{17}$ Another extension would be to let the model encompass supplements to labor income reflecting benefits like child allowances, day care subsidies and housing benefits. It may also be worthwhile to allow for individual differences in preferences and in the access to benefit systems. For instance, individuals may differ in their sensitivity to social norms and they may also have differing entitlements to transfer payments. Finally, it might be valuable to model a more realistic political process than the simple majority rule used in this paper; by instead studying political institutions such as representative democracy and political parties.

\footnotetext{
${ }^{17}$ It can be shown that if individuals have Cobb-Douglas preferences - and, as here, lack nonlabor incomes - then the present limitation to such a binary choice is not binding, see Sundén and Weibull [20].
} 


\section{Appendix}

\subsection{Proof of proposition 2}

Suppose $p=(t, T)$ is a balanced policy, and assume $t>0$ and $x=\xi(p)<\frac{1}{2}$. Individuals with wages exceeding the critical wage $w^{*}(t, T, x)$ then work and constitute a strict majority. Moreover, they would have higher utility in the zero-tax zero-transfer state $s^{o}=(0,0,0)$ than in the proposed state $s=(t, T, x)$, simply because $u\left(w_{i}\right)>u\left((1-t) w_{i}\right)$. Thus $p^{o}=(0,0)$ beats $p$. Hence, if $p$ is unbeatable and $t>0$, then $x \in\left[\frac{1}{2}, 1\right)$.

Suppose $p=(t, T)$ is balanced and unbeatable, $t>0$, and $x=\xi(p) \in\left[\frac{1}{2}, 1\right)$. Then individuals with wages below the critical wage $w^{*}(t, T, x)$ constitute a weak majority. Assume $x \notin \hat{X}$. Let $x^{\prime} \in \hat{X}$ and $p^{\prime}=\left(f\left(x^{\prime}\right), F\left(x^{\prime}\right)\right)$. Then the weak majority of transfer recipients under $p$ would have higher utility under $p^{\prime}$ than they have under $p$. For under $p$ their utility is $u(T)+\mu-v(x)$ while under $p^{\prime}$ the utility to any individual $i$ is

$$
\begin{aligned}
U_{i}\left(p^{\prime}\right) & =\max \left\{u\left(T^{\prime}\right)+\mu-v\left(x^{\prime}\right), u\left(\left(1-t^{\prime}\right) w_{i}\right)\right\} \\
& \geq u\left(T^{\prime}\right)+\mu-v\left(x^{\prime}\right)>u(T)+\mu-v(x)
\end{aligned}
$$

By continuity also some wage earners under $p$ (those with wages just above the critical wage) would have higher utility under $p^{\prime}$. Thus $p$ is beaten by $p^{\prime}$. Hence $x \in \hat{X} \cap\left[\frac{1}{2}, 1\right)$ if $p$ is unbeatable and $t>0$.

Suppose finally that $p=(t, T)$ is balanced and unbeatable, $t>0$, and $x \in$ $\hat{X} \cap\left[\frac{1}{2}, 1\right)$, but $x \neq \hat{x}$. Then the (possibly weak) majority of transfer recipients under $p$ would have at least the same utility under $\hat{p}$ as they have under $p$, since both $x$ and $\hat{x}$ belong to $\hat{X}$, and transfer recipients under $p$ may choose, under $\hat{p}$, to stay on the transfer. Moreover, the positive population share of workers under $p$ have higher utility in $\hat{p}$. Under $p$ the utility of a worker $i$ is $u\left([1-f(x)] w_{i}\right)$ while under $\hat{p}$ his utility is

$\max \left\{u(F(\hat{x}))+\mu-v(\hat{x}), u\left([1-f(\hat{x})] w_{i}\right)\right\} \geq u\left([1-f(\hat{x})] w_{i}\right)>u\left([1-f(x)] w_{i}\right)$.

Consequently $p$ is beaten by $\hat{p}$.

In sum: $x=\hat{x} \geq \frac{1}{2}$ if $p$ is balanced, unbeatable, and $t>0$.

\subsection{Proof of proposition 3}

First, suppose $u(\tilde{w}) \geq \beta$. Let $p^{o}$ be the zero-tax policy, and let $p^{\prime}=\left(t^{\prime}, T^{\prime}\right)$ be a balanced policy with $t^{\prime}>0$. Let $x^{\prime}=\xi\left(p^{\prime}\right)$. Clearly $p^{o}$ is not beaten by $p^{\prime}$ if $x^{\prime} \leq \frac{1}{2}$, since then the workers under $p^{\prime}$ constitute a (possibly weak) majority, and they pay a positive income tax in $s^{\prime}$. Assume $x^{\prime}>\frac{1}{2}$. The critical individual in $s^{\prime}$ earns pre-tax wage $w^{\prime}=\Phi^{-1}\left(x^{\prime}\right)>\tilde{w}$. All individuals with wages below $w^{\prime}$ prefer the transfer to work and $u\left(T^{\prime}\right)+\mu-v\left(x^{\prime}\right) \leq \beta$ by definition of $\beta$. However, 
$u\left(w^{\prime}\right)>u(\tilde{w}) \geq \beta$, a contradiction. Hence no such policy $p^{\prime}$ exists. Thus $p^{o}$ is unbeatable.

Second, suppose $u(\tilde{w})<\beta$. Let $p^{\prime}=\left(t^{\prime}, T^{\prime}\right)$ be balanced, with $x^{\prime}=\xi\left(p^{\prime}\right) \geq \frac{1}{2}$ and $u\left(T^{\prime}\right)+\mu-v\left(x^{\prime}\right)=\beta$ (such a policy $p^{\prime}$ exists by definition of $\beta$ ). Then the median wage earner is a transfer recipient in $s^{\prime}$ (since $u(\tilde{w})<\beta$ ) and has higher utility under $p^{\prime}$ than under $p^{o}$. This is also true for all individuals with lower wages, and, by continuity, also for individuals with wages slightly above $\tilde{w}$ so a strict majority prefers $p^{\prime}$ over $p^{o}$. Thus $p^{o}$ is not unbeatable.

Third, suppose $u(\tilde{w})>\beta$. In order to show that no policy $p \neq p^{o}$ is unbeatable, it suffices, by proposition 1 , to show that $\hat{p}$ is beaten by $p^{\circ}$. But this follows from the simple fact that if $u(\tilde{w})>\beta$ then the median wage earner, along with all individuals with higher wages, and, by continuity also some individuals with slightly lower wages, prefer policy $p^{o}$ to $\hat{p}$.

\subsection{Proof of proposition 4}

Suppose $\hat{x}>\frac{1}{2}$. First, suppose $u(\tilde{w})>\beta$. Then no policy other than $p^{o}$ is unbeatable, by proposition 3 . This proves the "only if" part of the statement. Second, suppose $u(\tilde{w}) \leq \beta$, and let $\hat{s}=(\hat{t}, \hat{T}, \hat{x})$. The utility to a transfer recipient in this macro state is $u(\hat{T})+\mu-v(\hat{x})=\beta$. In particular, the median wage earner has at least as high a utility in $\hat{s}$ as in the zero-tax macro state $s^{\circ}$, so all individuals with lower wages prefer $\hat{p}$ to $p^{o}$. Since $\hat{x}>\frac{1}{2}, p^{o}$ does not beat $\hat{p}$. Can any other policy $p$ beat $\hat{p}$ ? If $x=\xi(p) \notin \hat{X}$, then the transfer recipients in $\hat{s}$, a strict majority, receive more utility in $\hat{s}$ than in $s=(t, T, x)$, and hence $\hat{p}$ is not beaten by any such policy $p$. If instead $x=\xi(p) \in \hat{X}$, then the transfer recipients in $\hat{s}$ receive the same utility in $s$ as in $\hat{s}$. However, all tax payers in $\hat{s}$ receive more utility in $\hat{s}$ than in $s$. Thus, while transfer recipients in $\hat{s}$ are indifferent, all taxpayers in $\hat{s}$ prefer $\hat{p}$ to $p$, so $\hat{p}$ is not beaten by $p$. In sum: $\hat{p}$ is unbeatable if $u(\tilde{w}) \leq \beta$. 


\section{References}

[1] Akerlof, G. (1980), "A Theory of Social Custom, of Which Unemployment May Be One Consequence," Quarterly Journal of Economics, June, 749-775.

[2] Basu, K. (1987), "Monopoly, Quality uncertainty and 'status' goods, International Journal of Industrial Organization 5, 435-446.

[3] Becker, G. S. (1991), "A Note on Restaurant Pricing and Other Examples of Social Influences on Price", Journal of Political Economy 99, 1109-1116.

[4] Bernheim, B. D. (1994), "A Theory of Conformity", Journal of Political Economy 102, 841-877.

[5] Blomquist, N. S. (1993), "Interdependent Behavior and the Effect of Taxes", Journal of Public Economics 51, 211-218.

[6] Coleman, J.S. (1990), Foundations of Social Theory, Harvard University Press, Cambridge, Mass.

[7] Engbersen, G., Schuyt, K, Timmer, J. and F. Van Waarden (1993), Cultures of Unemployment: A Comparative Look at Long-Term Unemployment and Urban Poverty. Westview Press. Boulder, Colorado.

[8] Elster, J. (1989), The Cement of Society: A Study of Social Order, Cambridge University Press, Cambridge, UK.

[9] Gouldner, A.W. (1970), The Coming Crisis of Western Sociology, Equinox Books/Avon.

[10] Granovetter, M. (1978), "Threshold models of collective behavior", American Journal of Sociology 83 (6), 1420-43.

[11] Granovetter, M. and R. Soong (1983), "Threshold models of diffusion and collective behavior", Journal of Mathematical Sociology 9, 165-179.

[12] Granovetter M. and R. Soong (1986), "Threshold models of interpersonal effects in consumer demand", Journal of Behavior and Organization 7, 8399.

[13] Lindbeck, A. (1995), "Welfare State Disincentives with Endogenous Habits and Norms," Scandinavian Journal of Economics 97(4), 477-494.

[14] Lindbeck, A., S. Nyberg, and J.W. Weibull (1996), "Social Norms, the Welfare State, and Voting," The Industrial Institute for Economic and Social Research, WP 453. 
[15] Meltzer, A. H. and S. F. Richard (1981), "A Rational Theory of the Size of Government," Journal of Political Economy 5, 914-927.

[16] Moffitt, R. (1983), "An economic model of welfare stigma," American Economic Review 73, 1023-1035.

[17] Parsons, T. (1952), The Social System. Tavistock Publications, London.

[18] Roberts, K. (1977), "Voting over income tax schedules", Journal of Public Economics 8, 329-340.

[19] Schelling, T. (1971), "Dynamic models of segregation." Journal of Mathematical Sociology 1, 143-86.

[20] Sundén, D. and J. Weibull (1997), "Social norms and transfers", mimeo, IUI. 